\title{
Evolutionary conservation and molecular characteristics of repetitive sequences of Drosophila koepferae
}

\author{
IGNACIO MARÍN* \& ANTONIO FONTDEVILA \\ Departamento de Genética y Microbiología, Universidad Autónoma de Barcelona, 08193 Bellaterra, Barcelona, \\ Spain
}

\begin{abstract}
Thirteen middle repetitive DNA clones obtained from the genome of Drosophila koepferae have been tested for their evolutionary conservation in the other seven species of the buzzatii and martensis clusters (repleta group). All but two of these clones exhibit qualitatively similar patterns of hybridization in the eight species. The average interspecific hybridization signal is 85 per cent of that found intraspecifically, ranging from 73 to 93 per cent. Partial sequencing of six of these clones has shown sequences related to the retrotransposon Gypsy, first characterized in D. melanogaster, as well as to the Anopheles gambiae LINE elements $T 1 A g$ and $Q$. A fragment of a hitherto unknown, short inverted repeat transposable element has also been found. The evolutionary conservation of repetitive $D$. koepferae sequences seems to be related to the high proportion of simple DNA and inactive mobile elements in the genome of this species.
\end{abstract}

Keywords: Drosophila, Gypsy, LINE, repetitive DNA, repleta group.

\section{Introduction}

Middle repetitive sequences obtained from Drosophila melanogaster frequently show a low hybridization signal or are absent in some of its closest relatives, the other species of the melanogaster species subgroup (Young, 1979; Dowsett \& Young, 1982; Dowsett, 1983). These studies also demonstrated that mobile sequences are the main middle repetitive DNA component of the genome of $D$. melanogaster. Interestingly, the repetitive sequences obtained from $D$. simulans, a sibling of $D$. melanogaster, are much more conserved in the other melanogaster subgroup species, and the genome of $D$. simulans is characterized by a low number of mobile elements (Dowsett, 1983). This relationship between mobility and evolutionary instability, particularly loss of mobile sequences in closely related species, has been predicted theoretically and is well supported by experimental evidence. Provided there is a long evolutionary time, theoretical models predict frequent stochastic losses of mobile elements in finite populations, especially

*Correspondence: Department of Biological Sciences, Stanford University, Stanford, CA 94305, U.S.A. under certain conditions such as small population sizes, high excision rates, or generation of defective elements that compete with active ones (Charlesworth, 1985; Kaplan et al., 1985; Charlesworth \& Langley, 1989). In good agreement with these expectations, when 11 characterized mobile elements (10 from $D$. melanogaster and one from $D$. mauritiana, also a species of the melanogaster subgroup) were tested for their phylogenetic distribution in other species of the Drosophila genus, all showed a patchy distribution. Moreover, nine of these elements have a low hybridization signal in one, or frequently several, of the $D$. melanogaster subgroup species (Martin et al., 1983; Brookfield et al., 1984; Bucheton et al., 1986; Stacey et al., 1986; Silber et al., 1989; Daniels et al., 1990a,b; Mizrokhi \& Mazo, 1990; Maruyama \& Hartl, 1991).

In a previous study (Marín et al., 1992), we characterized the middle repetitive component of $D$. koepferae and $D$. buzzatii, two sibling species of the repleta group. Our results showed three interesting features. First, when hybridized against genomic DNA, a high number of clones showed a smeared pattern, similar to those produced by simple DNA sequences. These sequences are so abundant that the percentage of repetitive DNA in these two 
species is estimated to be higher than that found in any other species of the genus. Secondly, out of more than 100 clones tested, all but two showed similar hybridization patterns against the genomic DNA of both species. Finally, although all those clones which showed a pattern of hybridization against genomic DNA compatible with mobile elements (a limited number of bands with similar hybridization intensities) were tested, only two from $D$. koepferae and one from $D$. buzzatii showed a dispersed pattern of in situ hybridization on polytene chromosomes. The conclusion of our preliminary work was that the more abundant repetitive component of these species is quite different from that of $D$. melanogaster, and is probably simpler and more repetitive. Sequences related to mobile elements, with only a few exceptions, were predicted to be inactive and concentrated in the centromeres.

In this study we have tested for the evolutionary conservation of certain repetitive sequences of $D$. koepferae in other related species of the repleta group. In addition, by sequencing different fragments of a number of clones, we have recognized sequences with clear relationships with previously described mobile elements, the retrotransposon Gypsy of D. melanogaster (Marlor et al., 1986) and the nonviral retroposons $T 1 A g$ and $Q$, first characterized in Anopheles gambiae (Besansky, 1990; Besansky et al., 1994). A fragment of a new short inverted repeat mobile element has also been detected. Simple DNA sequences were also found, and we have an example of a scrambled arrangement of one of these sequences with a defective mobile element. These results help to clarify the differences between the repetitive components of $D$. koepferae and D. melanogaster.

\section{Materials and methods}

\section{Drosophila strains}

Strains from the eight species that form the martensis and buzzatii clusters (buzzatii complex, mulleri subgroup, repleta group; Ruíz \& Wasserman, 1993) were used. These will be referred to as follows. (a) buzzatii cluster: KO4 (D. koepferae, Vipos, Argentina); BSL (D. buzzatii, San Luis, Argentina); SD14 (D. serido, Cafarnaum, Brazil, Bowling Green stock no. 15081-1431.4); BM1 (D. borborema, Cafarnaum, Bowling Green stock no. 15081-1281.0). (b) martensis cluster: SM3 (D. starmeri, Curaçao, Netherland Antilles); VZ8 (D. venezolana, Curaçao); UN5 ( $D$. uniseta, La Boca, Venezuela); MA4 (D. martensis, Guaca, Venezuela).

\section{Characteristics of the clones}

Thirteen clones obtained from stock $\mathrm{KO} 4$ were used in this study. The sizes, patterns of hybridization against genomic DNA, and results of in situ hybridization on polytene chromosomes of 11 of these clones were described in Marín et al. (1992). The other two, cDk13 (4.6 kb) and cDk32 (6.1 kb) share their patterns of genomic DNA (one main band plus smeared hybridization) and in situ hybridization (one euchromatic band).

\section{Southern blot analysis}

Total genomic DNA from adult flies, obtained according to Piñol et al. (1988), was cut with HindIII, electrophoresed in 0.6 per cent agarose gels and transferred to Hybond-C extra nitrocellulose membranes (Amersham International, Bucks., U.K.). In each electrophoretic run, lanes were loaded with approximately the same amount of DNA $(3-5 \mu \mathrm{g})$. Preparation of the probes, hybridization and developing procedures were performed as described by Marín et al. (1992). The stringency of hybridization $(5 \times \mathrm{SSC}, 50$ per cent formamide, $\left.42^{\circ} \mathrm{C}\right)$ and posthybridization washes $(0.16 \times \mathrm{SSC}$, $50^{\circ} \mathrm{C}$ ) used allows detection of sequences with at least 80 per cent identity (supposing G-C content $=50$ per cent and a paired sequence length of $0.5 \mathrm{~kb}$; Wetmur, 1991).

\section{Quantification of the hybridization signals}

The intensity of the hybridization signals was measured using a Vinix (Vicom-VDP) image analysis system. Images from each lane were digitized using a Hamamatsu c2400/01 camera equipped with a Canon $35-70 \mathrm{~mm}$ macro zoom lens under constant lighting, converting them into an array of pixels, each with grey values ranging from 0 to 128 . For each lane, the average intensity per pixel, calculated after dividing the integrated hybridization intensity in that lane by its area, was corrected by subtracting the average intensity per pixel in a blank area of the same filter. The relative abundance of DNA hybridization (Dowsett \& Young, 1982; Dowsett, 1983) was calculated by dividing the average intensity per pixel in the experimental lane by that of the control lane (KO4 strain), after subtracting the background signal, and eventually correcting for differences in DNA quantity. DNA amounts were estimated by a similar procedure using Polaroid photographs of the ethidium bromide-stained electrophoresis gel taken before Southern transfer. Under our current system 
of quantification there was a linear relationship between DNA quantity and signal intensity in these gels up to a saturation value ( 75 grey intensity units/ pixel). Eighty-seven out of the 91 estimations (96 per cent) were included in the linear part of the curve.

\section{DNA sequencing and sequence analysis}

Plasmid pBluescript $\mathrm{KS}^{+}$(Stratagene) was used for subcloning procedures. High-quality supercoiled plasmid DNA for sequencing was obtained using Magic Minipreps columns (Promega, Madison, WI). Nucleotide sequences were determined by using the dideoxinucleotide chain-termination method (Sanger et al., 1977). For the determination of similarities and sequence alignments, the GCG software package (Genetics Computer Group, 1991) was used. The FASTA program was used for nucleotide searches against the GenBank and EMBL databases. The nucleotide sequences were translated in the six possible frames and the predicted proteins were compared with the PIR and Swiss-Prot databases using TFASTA. The BESTFIT and PILEUP programs were used for establishing the best alignments of the zones that gave positive results in the FASTA or TFASTA searches. Although most of the sequences were determined only in one strand, this does not affect any of the conclusions concerning the homologies found (they extend along hundreds of base pairs) or the activity of the copies of the elements analysed (we have considered inactive those sequences with large insertions or deletions).

\section{Results}

Most of the D. koepferae repetitive sequences tested are shared by the other seven species of the buzzatii and martensis clusters

Table 1 summarizes the estimations of the intensity of hybridization for the 13 clones. The average for all comparisons was $0.85 \pm 0.04$. Eleven of the 13 repetitive sequences, the exceptions being cDk210 and cDk254, showed similar overall patterns of hybridization in the eight species of the buzzatii and martensis clusters (some examples are shown in Fig. 1). Differences found for some species in the clones cDk2, cDk13 and cDk665 were quantitative, but their hybridization patterns were similar to that of $D$. koepferae. This result confirms our previous findings: out of more than 100 repetitive clones obtained from the siblings $D$. koepferae and $D$. buzzatii, only cDk210 and cDk254 revealed significant differences in hybridizations against genomic DNA of these two species (Marín et al., 1992). When our results are compared with those obtained for D. melanogaster (Dowsett, 1983), it can be seen that the distributions of the relative abundance of hybridization are qualitatively different (Fig. 2). When $D$. koepferae sequences were used as probes, only three out of 91 estimates ( 3.3 per cent) had

Table 1 Relative abundance of hybridization of the thirteen Drosophila koepferae clones in the other species of the buzzatii and martensis clusters. The control value for D. koepferae is 1.00. BSL, D. buzzatii; SD14, D. serido; BM1, D. borborema; $\mathrm{SM} 3$, D. starmeri; VZ8, D. venezolana; UN5, D. uniseta; MA4, D. martensis

\begin{tabular}{lccccccc}
\hline Clone & BSL & SD14 & BM1 & SM3 & VZ8 & UN5 & MA4 \\
\hline cDk2 & 1.03 & 1.20 & 0.98 & 0.56 & 1.29 & 0.53 & 0.28 \\
cDk13 & 0.67 & 0.37 & 0.51 & 0.37 & 0.24 & 0.50 & 0.49 \\
cDk27 & 0.68 & 0.87 & 0.63 & 0.79 & 0.90 & 0.89 & 0.92 \\
cDk32 & 0.96 & 0.84 & 0.83 & 1.01 & 1.06 & 0.81 & 0.81 \\
cDk72 & 0.89 & 0.67 & 0.75 & 1.01 & 1.15 & 1.38 & 1.32 \\
cDk210 & 0.25 & 0.65 & 0.66 & 0.60 & 0.58 & 0.48 & 0.59 \\
cDk254 & 0.10 & 0.19 & 0.54 & 0.49 & 0.49 & 0.41 & 0.01 \\
cDk265 & 0.72 & 0.71 & 0.71 & 0.66 & 0.64 & 0.81 & 0.86 \\
cDk270 & 0.80 & 0.96 & 1.12 & 0.83 & 0.67 & 0.66 & 0.48 \\
cDk271 & 1.68 & 1.80 & 1.91 & 0.71 & 1.23 & 1.39 & 1.13 \\
cDk323 & 1.31 & 1.01 & 1.76 & 0.73 & 1.54 & 1.85 & 1.73 \\
cDk596 & 1.11 & 1.09 & 1.01 & 1.27 & 1.38 & 1.16 & 1.31 \\
cDk665 & 0.77 & 1.01 & 0.73 & 0.48 & 0.39 & 0.41 & 0.29 \\
Means \pm SEM & $0.84 \pm 0.11$ & $0.87 \pm 0.11$ & $0.93 \pm 0.12$ & $0.73 \pm 0.07$ & $0.89 \pm 0.12$ & $0.87 \pm 0.13$ & $0.79 \pm 0.14$ \\
\hline
\end{tabular}



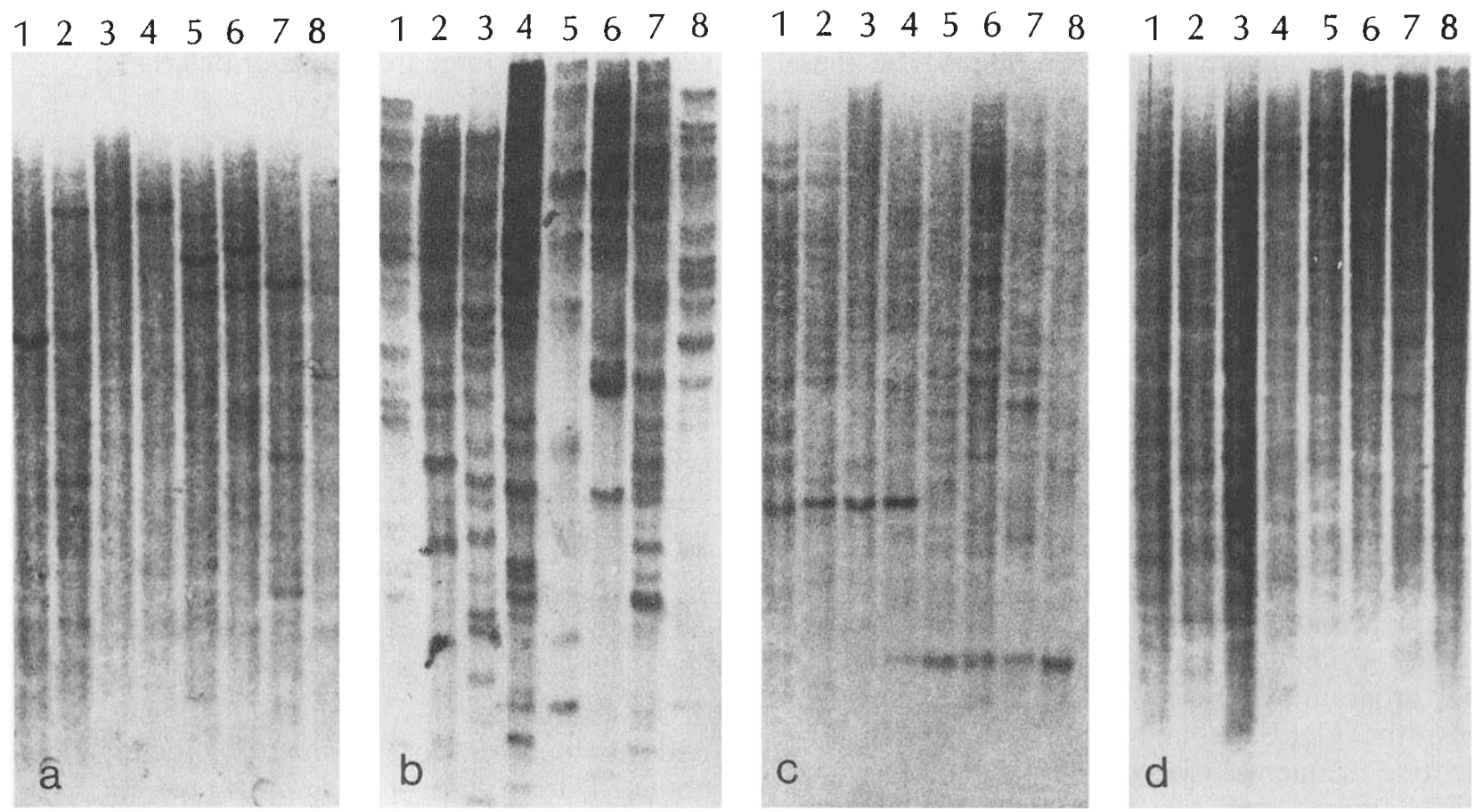

Fig. 1 Examples of hybridization patterns of Drosophila koepferae repetitive sequences in the species of the buzzatii and martensis clusters. (a) cDk596; (b) cDk323; (c) cDk2; (d) cDk27. Lanes: 1, KO4 (D. koepferae); 2, BSL (D. buzzatii); 3 , SD14 (D. serido); 4, BM1 (D. borborema); 5, SM3 (D. starmeri); 6, VZ8 (D. venezolana); 7, UN5 (D. uniseta); 8, MA4 (D. martensis).

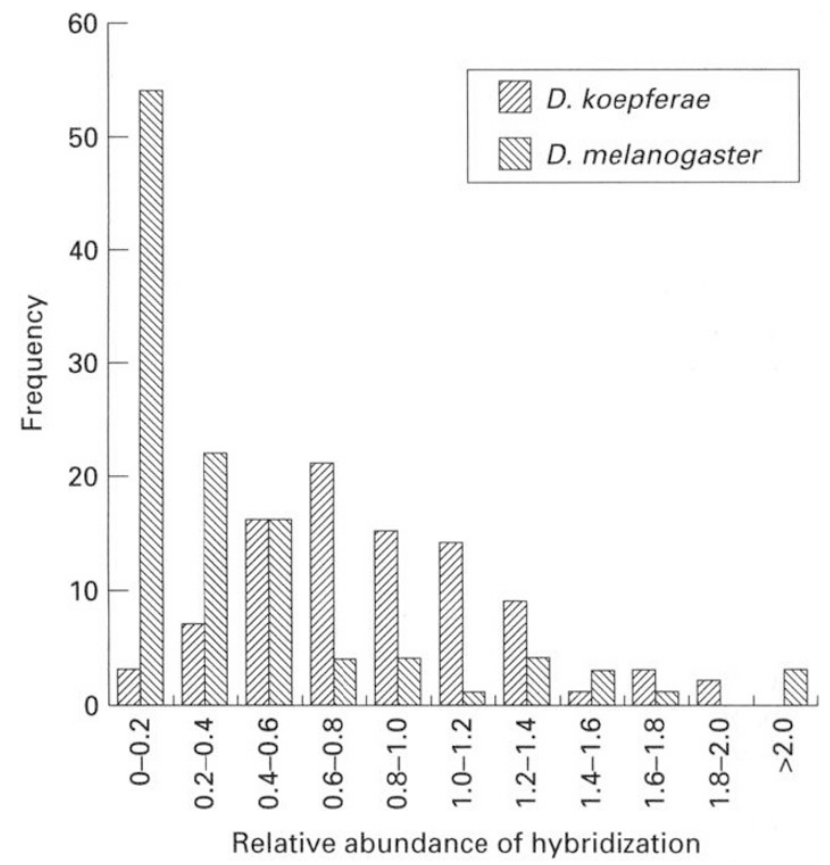

Fig. 2 Distributions of the values of the relative abundance of hybridization. Drosophila koepferae: 91 comparisons (this study). D. melanogaster: 112 comparisons (Dowsett, 1983). values lower than 0.20 . However, using $D$. melanogaster repetitive sequences, this percentage was 48.2 per cent (54 out of 112 comparisons).

\section{Partial sequencing of selected clones reveals fragments of three different mobile elements}

The sequence of one of the clones studied here (cDk27, $0.7 \mathrm{~kb}$ ) was shown by Marín et al. (1992). It carries two stretches of low-complexity DNA. In this study we have sequenced $8.4 \mathrm{~kb}$, including different fragments of the other six clones, accounting for 37 per cent of their length. These clones were chosen because their pattern of hybridization against genomic DNA and/or their in situ pattern on polytene chromosomes suggested that they could carry mobile sequences. Our strategy has been to sample for the types of sequences in these clones by sequencing randomly several zones, starting from some of the available restriction sites. A summary of these results is as follows.

$c D k 2(4.5 \mathrm{~kb})$ In this centromere-hybridizing clone, $1.9 \mathrm{~kb}$ have been sequenced. Two different parts have shown similarity to mobile elements that have

(C) The Genetical Society of Great Britain, Heredity, 76, 355-366. 
been described previously. The predicted protein for one zone is related to those of the $T 1 A g$ and $Q$ nonviral retroposons (Besansky, 1990; Besansky et al., 1994). In particular, a fragment related to its reverse transcriptase has been detected. A second interesting region shows significant similarity to the Gypsy retrotransposon as well as to other Gypsyrelated sequences. Inside this sequence a $350 \mathrm{bp}$ simple DNA stretch is found, consisting in partially degenerate TAA repeats (see below).

$c D k 210(1.8 \mathrm{~kb}) \quad$ Marín et al. (1992) found that this clone has a dispersed repetitive sequence with variable cytological positions in different $D$. koepferae stocks. This clone was used as a probe to obtain three homologous recombinant phages and the dispersed sequence has been characterized. It consists of a new short inverted repeat mobile element that we have named Gandalf (Marín \& Fontdevila, 1995). Sequencing of cDk210 showed that it carries $0.5 \mathrm{~kb}$ of the putative $3^{\prime}$ end of this element.

$c D k 254(2.6 \mathrm{~kb}) \quad$ Approximately $2.0 \mathrm{~kb}$ of this clone has been sequenced. cDk254 hybridizes predominantly on the telomeric regions (Marín et al., 1992). Comparisons with sequence data banks did not show significant DNA similarities.

$c D k 270(4.9 \mathrm{~kb})$ This clone bears a dispersed repetitive sequence and also hybridizes in the centromeres (Marín et al., 1992). $1.0 \mathrm{~kb}$ have been sequenced. Similarity to the $T 1 A g$ and $Q$ elements has been observed, corresponding to the same regions found in $\mathrm{cDk} 2$.

$c D k 323(5.1 \mathrm{~kb})$ This clone is mainly centromeric, although a few euchromatic bands have been found in some stocks (Marín et al., 1992). $1.4 \mathrm{~kb}$ have been sequenced. A very high similarity to the reverse transcriptase of Gypsy and other Gypsy-type elements has been found.

$c D k 665(3.9 \mathrm{~kb})$ Of this clone, $1.5 \mathrm{~kb}$ has been sequenced without significant findings.

\section{Gypsy-related defective sequences in the genome of $\mathrm{D}$. koepferae}

$\mathrm{cDk} 2$ and $\mathrm{cDk} 323$ bear sequences closely related to the Gypsy element. Similarity is found both at the DNA and protein levels. Comparisons with the $D$. melanogaster and $D$. virilis elements show that the Gypsy-related sequence of cDk2 is divided into two fragments (A and B) by a $350 \mathrm{bp}$ sequence of unknown origin, composed mainly of degenerate (TAA) ${ }_{n}$ repeats (Fig. 3 ). Regions A and B of cDk2 are $300 \mathrm{bp}$ apart in the Gypsy sequences of $D$. melanogaster and $D$. virilis, in the same order as they are found in cDk2. Clearly they comprise a single Gypsy-like sequence in which this 300 bp inner fragment has been substituted by the TAA repeat. There are no signs of any (TAA) ${ }_{n}$ sequence in the homologous zones of the $D$. melanogaster or $D$. virilis Gypsy elements.

Database searches revealed that region A (265 bp) shows significant nucleotide identity with five different sequences; that is, the Gypsy elements of D. melanogaster and D. virilis (Marlor et al., 1986; Mizrokhi \& Mazo, 1991), a third sequence (accession number X15707) found in association with different rRNAs in a D. melanogaster clone (V. A. Kokoza, N. G. Kholodilov and I. B. Rogozin, unpublished data) and two sequences obtained from the $\mathrm{Y}$ chromosome of $D$. hydei (accession numbers X74538 and X74539; Hochstenbach et al., 1994). Region B ( $357 \mathrm{bp}$ ) has been found to be related to four of these five sequences, and to another one, a piece of a Gypsy-related element inserted in a Micropia retrotransposon of $D$. hydei (clone DhMiF8; accession number X13305; Lankenau et al., 1990). The protein alignments of these two regions are shown in Fig. 4. The Gypsy sequences of cDk2 are similarly related to all these sequences, irrespective of the phylogenetic proximity of their host species. Their nucleotide identities range between 50 and 60 per cent. These results contrast with the fact that the active elements of $D$. melanogaster and $D$. virilis show a 75 per cent nucleotide identity in the same zones. At the protein level, the similarity between the active elements (over 80 per cent, identity over 75 per cent) is also higher than the values for the cDk2 sequence (identity 40 per cent, similarity 60 per cent).

Two features of the Gypsy-related sequence of $\mathrm{cDk} 2$ are worthy of mention. First, this sequence corresponds to the env-like gene characteristic of the Gypsy-type retrotransposons (Smith \& Corces, 1991). This gene, which codes for the envelope protein in retroviruses, is known to change rapidly (McClure et al., 1988). No other characterized retrotransposon has shown homology with the env-related sequence of $\mathrm{cDk} 2$. A second important point is that the cDk2 Gypsy-related sequence is clearly nonfunctional. Not only does it have a $350 \mathrm{bp}$ insertion, but also frameshifts have been found in the predicted coding region. A similar situation is found in the Gypsy-related sequence in $\mathrm{cDk} 323$. The character- 
ized sequence is related to the carboxyl terminal end of the Gypsy reverse transcriptase enzyme. This sequence is more conserved among elements than those found in $\mathrm{cDk} 2$, as expected for a protein that is much more functionally constrained than the env-related sequences. Fig. 5 shows the best alignments of $\mathrm{cDk} 323$, indicating the domains $4-7$ of the reverse transcriptase enzyme, as defined by Xiong \&

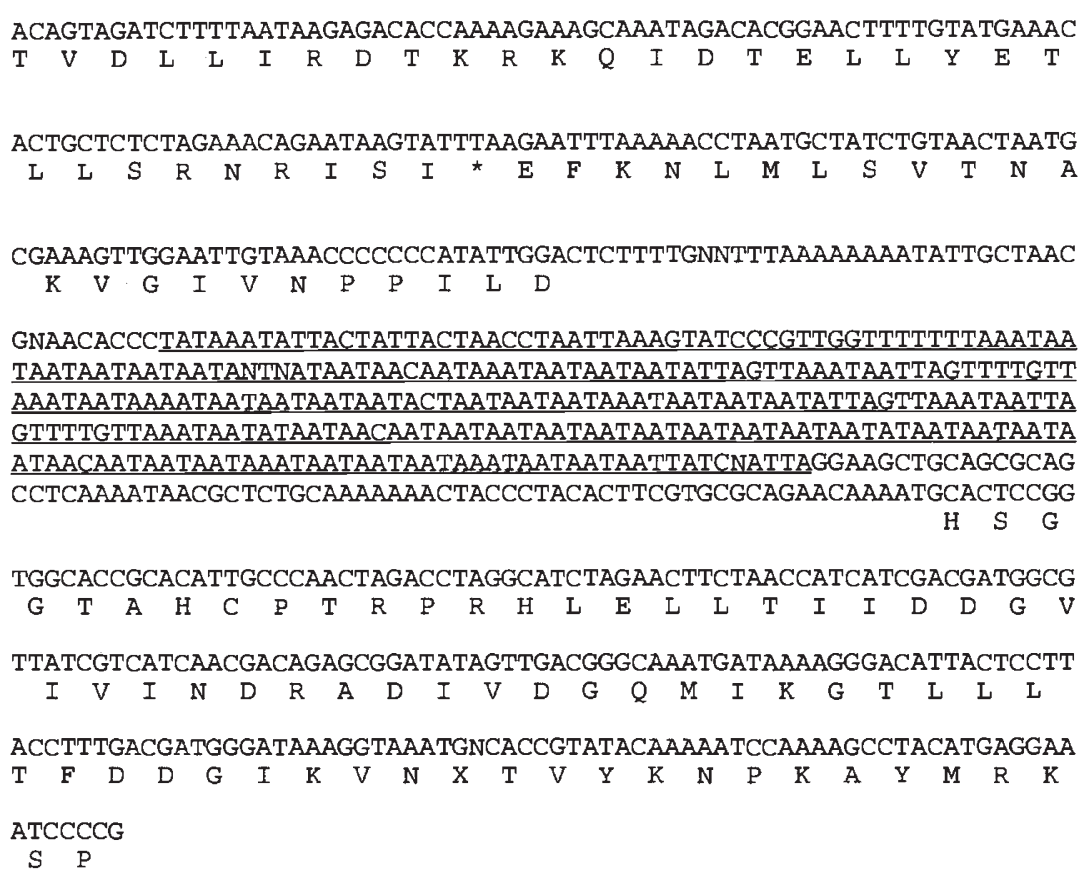

REGION A

CDK2

GYPSY1

GYPSY2

X15707

X74538

$\times 74539$

CONSENSUS

\section{REGION}

$\mathrm{CDk} 2$

GYPSY1

GYPSY2

$\times 15707$

$\times 13305$

$\times 74538$

CONSENSUS

CDK2

GYPSY 1

GYPSY2

X15707

$\mathrm{X} 13305$

$\times 74538$

CONSENSUS
TVDLLIRDTKRKOIDTELL YETLLSRNR I IXEFKALMLSVTNAKVGIVNPPILD TI NKVINARKGDLVDTPHL YEALLARNRMLSTE IONL I LT I TLVRSNI INPTILD TINK I ISSRKGDLVDTPHLFETLLARNRI LNSEIQNLVLT I TLARASIVNPTILD NRQFNFEI SQT SQIDTGHL YETLLARNRMFMAELQNLMLA I TLAK INIVSSNILD TVNEI LKAWRA IQIDTSHL YETLLARNRMLTLE I ENL IVA I TLAT ANI INPVI IN TINKI ISSRKGDLVDTPHL FETLLARNR ILNTE IWNIML T I TLAKGNIVAPT ILD

T-N--I---K----DT-HLYETLLARNR-L--EI-NL-L-ITLAK-NIVNP-ILD

HSGGTAHCPTRPRHLELLTIIDDGVIVIND-- -RADIVDG--- OMIKGTLLLTFD HAGNAAQCHTQPSHL RE INPVDDGVVIINEAAAHVSTDGS-PETLIEGTYLVTFE HA GNI ANCH TQP SHLKAIMPVDDGVVVINEATAHVRTDDD-AEVTVSGTFL I TFE HAGGVAHCRVOESDLHPI TYVDEGII I INDRSAKVRVDNGY / KSWTHGTHL I TFD QAGGTARCHTQPSHLKPITVVDDGIVVVNESPARISTDDS-PEIAVN----VTFE HAGK TAHCNTQPNELAVVTLVDDGILVLNDS PARVTIDDG-PENTITGTYLVTFD

HAG- -A-C-TQP SHL--IT-VDDG--VIN---A-V--DD---E-D--GT-L-TF-

DGIKVNXTVYKNPRAYMRKSP

RTAT INGSEFVN----LRKTI

RSAT INGTEFVN----LRKSI

KQATINDTPPSSTTITPRREL

RTAFINGSKYVN----RREIL

RFAYVNGTRFTNNNGIVKMIP

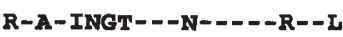

Fig. 3 Regions related to Gypsy in the Drosophila koepferae clone cDk2: the two zones with similarity (referred to as regions $\mathrm{A}$ and $\mathrm{B}$ in the text) and their predicted proteins. They are separated by a $300 \mathrm{bp}$ sequence with numerous TAA repeats (underlined).
Fig. 4 Protein sequence similarity among the predicted product of the Drosophila koepferae clone $\mathrm{cDk} 2$ and Gypsy sequences. Bold letters represent amino acids identical to those in cDk2. Some gaps (-) and frameshifts (/) have been added to improve the alignments. The consensus sequence includes those positions where at least four sequences have the same amino acid. GYPSY1, D. melanogaster Gypsy element; GYPSY2, D. virilis Gypsy element; X15707, D. melanogaster Gypsy-related sequence; $\mathrm{X} 13305, \mathrm{X} 74538$ and X74539, D. hydei Gypsy-related sequences (see text). 
CDk323

GYPSY1

GYPSY2

$\times 74538$

$\times 74539$

$\mathrm{X} 15707$

CONSENSUS
EFCRLPFGLTEAPSIFQRSI-DVLREQIGKI CYVYVDDVII---- -EFCRLPFGLRNASSIFQRALDDVLREQIGRI CYVYVDDVIIFSENESD BFCRLPFGLRNASSIFORAIDDILREHICKI CFVYVDDVIIFSKNETE EFCRLPFRSRPDGSIFORTIDDVLRARIGKT CYVYVDDVIIFSKSELD FFCRLPFGLRNASGIFQRA/DDIIREHIGKI CFVYVDDVIIFSKNERE

EFCRLPFGLRNA-BIFQR-DD-LRE-IGKI C-VYVDDVIIFS--E--
Fig. 5 Alignment of the predicted product of the sequence found in the Drosophila koepferae clone cDk323 with the retrotranscriptases of the Gypsy-related sequences (domains 4-7 according to Xiong \& Eickbush, 1990). Consensus sequence obtained as in Fig. 4. Bold letters represent amino acids identical to those found in cDk323. Gaps (-) and frameshifts (/) have been included to improve alignment. GYPSY1, D. melanogaster Gypsy element; GYPSY2, D. virilis Gypsy element; X74538, X74539, D. hydei Gypsy-related sequences; X15707, D. melanogaster Gypsyrelated sequences (see text).

CDk323
GYPSY1
GYPSY2
X74538
X74539
X15707

CONSENSUS
Domain 7
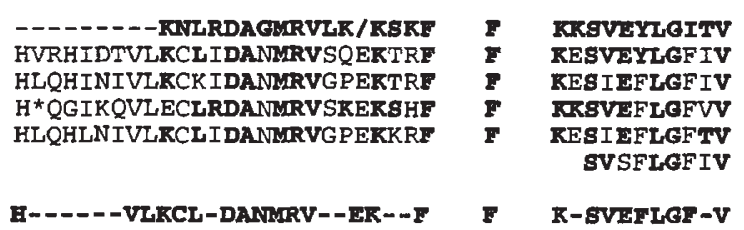

CDk323 GYPSY1 GYPSY2

$\times 74538$

$\times 74539$

$\mathrm{x} 15707$

CONSENSUS
CRGGIQTSADKVRAIKDEQP PKTLFNLRSFLGXXSYYRCF IAGFAKIA SKDGTKSDPEKVKAIQEYPEPDCVYKVRSFLGLASYYRVFIKDFAAIA TKDGATSDPKKVKAIQEF PEPKNVYSVRgFIGLANYYRVFIKDFAAIA SSSGTRT SOEKVHAIKNFAEPTNUFAVR SFLGLASYYRCFVRDFAAIA TKDGATSDPKKVKAFQEFPKPKSVHSVRSELGLA SYYRVF IKDFAAIV TCNGATTDPEKVHAIKEFPEPKSVFEIRSFLGLA SYYRRFIKGFSKLA
Eickbush (1990). As this figure shows, cDk323 domains 5 and 6 have been partially deleted. Moreover, a frameshift mutation is found in domain 6 . As it occurred in cDk2, the Gypsy-related sequence of cDk323 is defective. cDk323 is similarly related to the $D$. melanogaster and $D$. virilis elements (average nucleotide identities, 62 per cent; average protein similarities, 77 per cent), whereas the comparison between these active elements shows values of 75 per cent nucleotide identity and 90 per cent protein similarity in the same zones.

\section{D. koepferae has sequences related to T1Ag and $\mathrm{Q}$, two Anopheles gambiae nonviral retroposons}

Similar sequences have been found in clones cDk2 and cDk270. Database searches have shown that the putative proteins encoded by these sequences are related to those of the elements $T 1 A g$ and $Q$, two nonviral retroposons first found in the very distantly related dipteran Anopheles gambiae (Besansky, 1990; Besansky et al., 1994). However, DNA identities are below 50 per cent in the whole sequences (58 per cent in the best aligned regions).

Two different stretches showing similarity to these elements have been sequenced for each clone (Fig. 6). There was no a priori reason for expecting any similarity between these clones, because their restriction maps are completely different. In fact, the nucleotide sequences in these clones are only 82 per cent identical in the determined zones. The cDk2 and cDk270 sequenced zones most probably correspond to (1) region $\mathrm{A}$ : a zone of unknown function typical of some nonviral retroposons (a low similarity has been found with the $D$. melanogaster elements $F, G, D o c$ and Jockey, and especially with the Aedes aegypti element Juan-A; data not shown) placed at the beginning of the second open reading frame of these elements, amino terminally to the sequences related with the capsid proteins of retroviruses (McClure, 1991) and (2) region $B$ : the ' $Z$ ' region, immediately amino terminal to the reverse transcriptase (McClure, 1991), together with the first, and in $\mathrm{cDk} 2$, the second domains of this protein (Xiong \& Eickbush, 1990). As happened in the case of the env-related proteins, selective constraints acting on these regions are expected to be rare. Protein comparisons have shown that $\mathrm{cDk} 2$ and cDk270 are likewise related to $T 1 A g$ and $Q$ (cDk2/T1Ag, 32 per cent identity and 78 per cent similarity; cDk2/Q, 34 per cent identity and 75 per cent similarity; cDk270/T1Ag, 31 per cent identity and 79 per cent similarity; cDk270/Q, 36 per cent identity and 78 per cent similarity). Comparisons between these sequences established that region $B$ of $\mathrm{cDk} 270$ has a short deletion including part of the 


\section{REGION A}

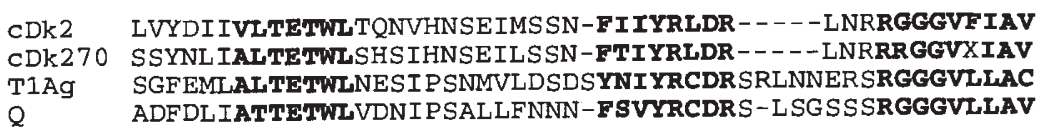

REGION B

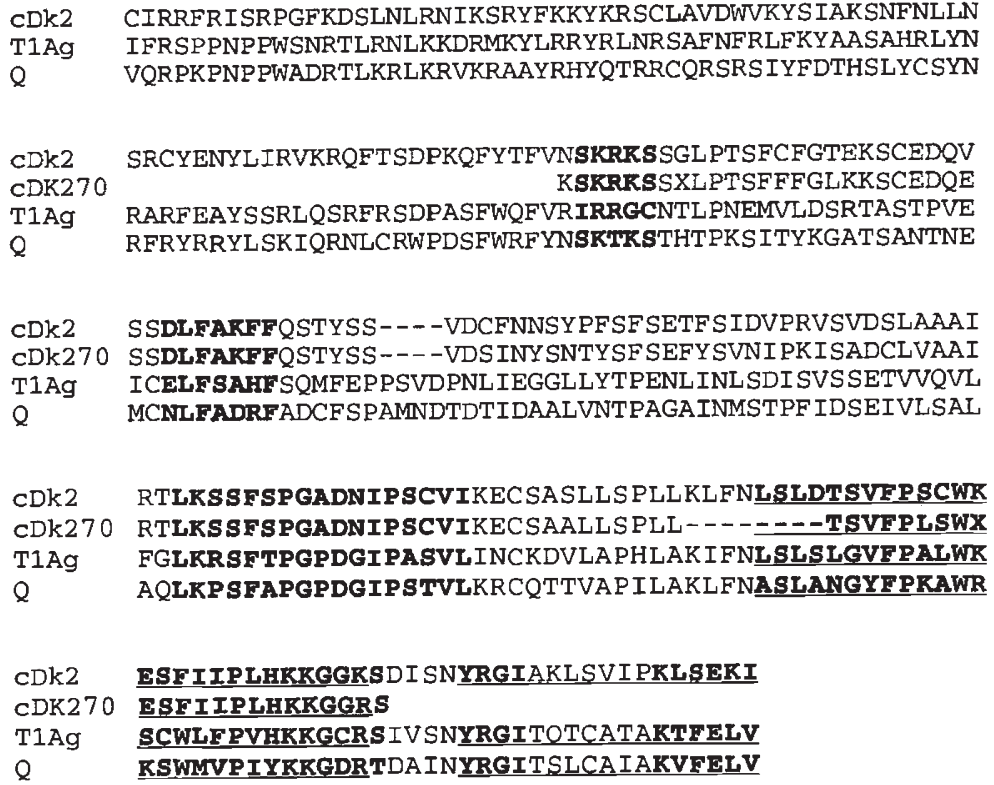

Fig. 6 Alignment of the $T 1 \mathrm{Ag} / \mathrm{Q}$ related sequences found in the Drosophila koepferae clones $\mathrm{cDk} 2$ and $\mathrm{cDk} 270$ with the sequences of the Anopheles gambiae elements. Bold: blocks of conserved amino acids. The two zones underlined in region $\mathrm{B}$ correspond to domains 1 and 2 of the reverse transcriptase (Xiong \& Eickbush, 1990). first domain of the reverse transcriptase. This deletion includes a frameshift, so, most probably, the sequence in $\mathrm{cDk} 270$ is not functional. The sequence determined in cDk2 does not allow us to eliminate the possibility that this clone contains part of a functional mobile element.

\section{Discussion}

\section{Conservation of $\mathrm{D}$. koepferae repetitive sequences}

Thirteen clones, obtained from an Argentinian stock of D. koepferae, were selected in order to test whether the repetitive sequences of this species are evolutionarily conserved in its closest relatives, in a study parallel to that of Dowsett (1983) on the melanogaster subgroup. No other groups have been similarly studied. A few sequences from $D$. subobscura (Felger \& Sperlich, 1989), D. algonquin (Hey, 1989 ) and $D$. virilis (Riede et al., 1983) have been tested in related species, but their degree of conservation was not analysed. Our results differ qualitatively from those found for $D$. melanogaster and its relatives (Fig. 2). It is difficult to find a single $D$. melanogaster repetitive sequence shared by all the melanogaster subgroup species, and the average intensity of interspecies hybridization is $0.39 \pm 0.05$ for D. melanogaster clones (Dowsett, 1983). On the other hand, most of the D. koepferae repetitive sequences are present in all of its closest relatives (average hybridization signal $0.85 \pm 0.04$ ).

This result can be explained in two ways. It may be argued that (i) the melanogaster subgroup species are less inter-related than the species of our study, and (ii) the proportions of the different classes of repetitive DNA in the two sets of species are different. The first possibility is unlikely, according to genetic distances as measured by allozymic differentiation. Cariou (1987) showed that Nei's genetic distances among the eight species of the melanogaster subgroup range from 0.28 (simulans-sechellia) to 1.74 (melanogaster-erecta). A similar study with the eight species of the buzzatii and martensis clusters produced estimates of Nei's distances ranging from 0.43 (starmeri-venezolana) to 2.02 (buzzatiivenezolana) (Sánchez, 1986; Fontdevila et al., 1988). These data suggest than both clusters split several million years ago. Moreover, differentiation among 
species within a given cluster is also high, suggesting that speciation events are ancient (Sánchez, 1986; Fontdevila et al., 1988). However, we have only exceptionally found reductions in the hybridization signals, not only in species of the buzzatii cluster but also in those of the martensis cluster, a result difficult to reconcile with their phylogenetic relationships.

The most likely explanation is therefore that there are differences in the molecular properties of the repetitive sequences. The analysed clones can be classified into two main groups. Clones cDk13, cDk32, cDk72, cDk265 and cDk596 are representative of sequences that show characteristic 'smeared' hybridization patterns against genomic DNA (see for example, Fig. 1a). These sequences are very frequent both in $D$. koepferae and in its sibling species $D$. buzzatii, accounting for 80 per cent of the repetitive clones obtained (Marín et al., 1992). These clones most likely bear simple DNA sequences, that would explain both their patterns of hybridization and their evolutionary conservation (Hamada et al., 1982; Tautz \& Renz, 1984; Morris et al., 1986; Tautz et al., 1986; Huijser et al., 1987; Pardue et al., 1987). Stretches of simple DNA sequences are frequent in our clones. We have found them in cDk27 (Marín et al., 1992) and $\mathrm{cDk} 2$ (Fig. 3), as well as finding in other clones near copies of the Gandalf element (Marín \& Fontdevila, 1995, and unpublished data).

A second group is formed by the other eight clones: cDk2, cDk27, cDk210, cDk254, cDk270, cDk271, cDk323 and cDk665. These clones were selected because they were the only candidates to bear mobile elements among more than $100 \mathrm{D}$. koepferae clones analysed by Marín et al. (1992). As expected, fragments of mobile element-related sequences have been recognized in $\mathrm{cDk} 2, \mathrm{cDk} 210$, cDk270 and cDk323. However, only in cDk210 have we been able to detect part of an active element (Marín \& Fontdevila, 1995). With the possible exception of the $T 1 \mathrm{Ag} / \mathrm{Q}$-related sequence in $\mathrm{cDk} 2$, the transposable element-related sequences found until now in cDk2, cDk270 and cDk323 are unambiguously inactive. They are structurally abnormal, suffering deletions and frameshifts in their coding regions. More work is needed in order to test whether active $T 1 A g / Q$-related sequences exist in $D$. koepferae. However, the centromeric hybridization of $\mathrm{cDk} 2$ suggests that this is unlikely. Although $\mathrm{cDk} 270$ also carries $T 1 A g / Q$-related sequences, its dispersed pattern on polytene chromosomes, different from that of $\mathrm{cDk} 2$, can be explained by the combination in this clone of two or more repetitive sequences, with at least one of them (other than the $T 1 \mathrm{Ag} / Q$ sequence and which has still not been characterized) being an active transposable element. Because our sequencing analysis has been partial and as difficulties exist in detecting zones (as long-terminal repeats, as well as other noncoding or rapidly evolving coding regions) or even types of mobile elements (as short inverted repeat elements), it is likely that other sequences derived from transposons exist in these clones.

The centromeric origin of most of these clones is important for an understanding of their evolutionary conservation. Drosophila pericentromeric $\beta$-heterochromatin is known to be formed by a complex mixture of repetitive sequences, interspersed with some single copy DNA (Healy et al., 1988; Miklos et al., 1988; Vaury et al., 1989; Shevelyov et al., 1989; Devlin et al., 1990; Russell et al., 1992). Thus, centromere-derived clones can bear several different repetitive sequences, so their combined signals will be detected. Our best characterized centromeric clone, $\mathrm{cDk} 2$, is an example of this type of arrangement. It contains at least two transposon-related sequences and one simple DNA sequence. Because of these findings, we favour the hypothesis that the apparent evolutionary stability of the D. koepferae repetitive sequences is related to the low number of active transposable elements in this species. This is a similar to what probably explains the higher relative abundance of hybridization of $D$. simulans repetitive sequences $(0.61 \pm 0.09)$, when compared to those of $D$. melanogaster $(0.39 \pm 0.05)$ in the same species of the melanogaster subgroup (Dowsett, 1983). Dowsett \& Young (1982) found that D. simulans repetitive clones came mainly from the centromeric regions.

\section{Gypsy- and T1Ag/Q-related sequences in D. koepferae: common origin or horizontal transfer?}

Sequences that hybridize with the Gypsy element have been found in a large number of Drosophila species, showing one of the more extended distributions among the mobile elements tested in the genus (Stacey et al., 1986). So far, Gypsy sequences have been detected in four species of the repleta group; $D$. repleta, D. mercatorum, D. mulleri (Stacey et al., 1986) and D. hydei (Lankenau et al., 1990; Hochstenbach et al., 1994). Drosophila mulleri is the closest relative to D. koepferae (Wasserman, 1992). In that species, Gypsy-related sequences are detected only under low stringency conditions when the Gyspy element of $D$. melanogaster is used as a probe (Stacey et al., 1986). Our D. koepferae Gypsyrelated nucleotide sequences are $50-60$ per cent 
identical to D. melanogaster Gypsy, in good agreement with these data.

The relationships among the Gypsy sequences found so far are interesting. The pattern that emerges is that active copies (in D. melanogaster and D. virilis) are quite similar, much more so than the inactive copies found $(\mathrm{cDk} 2$ and $\mathrm{cDk} 323$ sequences, X15707 sequence of D. melanogaster and sequences characterized in $D$. hydei), irrespective of the phylogenetic relationships among their host species. Horizontal transmission may be invoked to explain the similarity among active copies (Mizrokhi \& Mazo, 1991). Recent evidence suggesting that Gypsy may behave as an infectious virus (Kim et al., 1994; Song et al., 1994) makes this hypothesis very attractive. The characterization of active elements in other species will eventually explain the evolutionary history of this element.

The finding of sequences related to the $T 1 \mathrm{Ag}$ and $Q$ elements can be interpreted as evidence that they are ancient components of the genome of these species or, alternatively, as a new suggestion that horizontal transmission is an unexpectedly frequent phenomenon. Data on sequences related to known Drosophila transposons in other insect species are quickly accumulating. First, sequences related to $P$ and mariner elements, both of the DNA-transposing type, were found in Scaptomyza and Zaprionus, respectively, two genera closely related to Drosophila (Daniels et al., 1990b; Maruyama \& Hartl, 1991). After that, sequences related to mariner were first found in the Lepidopteran Hialophora cecropia (Lindholm et al., 1991) and later in a large number of other insects (Robertson, 1993). P-related sequences have also been found in Lucilia cuprina (the blowfly) (Perkins \& Howells, 1992). In parallel studies on retrotransposable elements, Martin et al. (1983) found hybridization signals of two retrotransposons, copia and 412, in the Zaprionus vittiger genome. Finally, a report of sequences related to the D. melanogaster Jockey nonviral retroposon in the species Apis mellifera and Bombyx mori has appeared, although this evidence is also indirect, obtained by hybridizations with a $D$. melanogaster probe (Kimura et al., 1993). Our study is the second direct demonstration, by means of sequencing, of retrotransposable sequences found in very distant species, after the finding of related $R 1$ and $R 2$ elements (ribosomal DNA insertions) in $B$. mori and D. melanogaster (Burke et al., 1987; Jacubczak et al., 1990) and later in other insect species (Jacubczak et al., 1991). Although the low similarity at the nucleotide level is compatible with an old origin of both sequences, the protein similarities (75-79 per cent, identities ranging from 31 to 36 per cent) are quite high, if the fact that the sequenced regions are expected to be diverging rapidly is taken into account. Some proteins have been found to be divergent to this extent when the homologous genes of species of the Drosophila genus are compared (O'Neil \& Belote, 1992). Only a detailed study of the phylogenetic distribution and the similarity of $T 1 A g$-related sequences may clarify whether horizontal transfer is a plausible explanation.

\section{Acknowledgements}

We thank Moritz Benado, Danko Brncic, Hugo Cerda, William J. Etges, Esteban Hasson, William B. Heed, Horacio Naveira, Alfredo Ruíz, Pilar Suyo, Jaime Vásquez and Mauro Santos for their help in several collections that provided some of the stocks used. We are also grateful to the National Drosophila Species Resource Center at Bowling Green State University which provided us with several stocks. We also thank Joan Masoliver (Centre de Tractament d'Imatges, U. A. B.), who provided the programs and technical support for the estimation of the relative abundance of hybridization, and Anuranjan Anand, for his comments on the final version of the manuscript. This work was supported by Spanish Dirección General de Investigación Científica y Técnica grants nos PB86/0064 and PB90/0711 to A.F. and by a predoctoral fellowship from the Generalitat de Catalunya to I.M.

\section{References}

BESANSKY, N. J. 1990. A retrotransposable element from the mosquito Anopheles gambiae. Mol. Cell. Biol., 10, $863-871$.

BESANSKY, N. J, BEDELL, J. A. AND MUKABAYIRE, O. 1994. $Q$ : A new retrotransposon from the mosquito Anopheles gambiae. Insect Mol. Biol., 3, 49-56.

BROOKFIELD, J. F. Y., MONTGOMERY, E. AND LANGLEY, C. H. 1984. Apparent absence of transposable elements related to the $\mathrm{P}$ elements of $D$. melanogaster in other species of Drosophila. Nature, 310, 330-332.

BUCHETON, A., SIMONELIG, M., VAURY, C. AND CROZATIER, M. 1986. Sequences similar to the I transposable element involved in I-R hybrid dysgenesis in $D$. melanogaster occur in other Drosophila species. Nature, 322, 650-652.

BURKe, w. D., CAlalang, C. C. AND EICKBush, T. H. 1987. The site-specific ribosomal insertion element type II of Bombyx mori (R2Bm) contains the coding sequence for a reverse transcriptase-like enzyme. $\mathrm{Mol}$. Cell. Biol., 7, 2221-2230.

CARIOU, M. L. 1987. Biochemical phylogeny of the eight species in the Drosophila melanogaster subgroup, includ-

(c) The Genetical Society of Great Britain, Heredity, 76, 355-366. 
ing $D$. sechellia and D. orena. Genet. Res., 50, 181-185. CHARLESWORTH, B. 1985. The population genetics of transposable elements. In: Ohta, T. and Aoki K. (eds) Population Genetics and Molecular Evolution, pp. 213-232. Japan Sci. Soc. Press/Springer-Verlag, Tokyo.

CHARLESWORTH, B. AND LANGLEY, C. H. 1989. The population biology of Drosophila transposable elements. Ann. Rev. Genet., 23, 251-287.

DANIElS, S. B., CHOVNIK, A. AND BOUSSY, I. A. 1990a. Distribution of hobo transposable elements in the genus Drosophila. Mol. Biol. Evol., 7, 589-606.

DANiels, s. B., PETERSON, K. R., STRAUSBAUGH, L. D., KIDWELL, M. G. AND CHOVNICK, A. 1990b. Evidence for horizontal transmission of the $P$ transposable element between Drosophila species. Genetics, 124, 339-355.

DEVLIN, R. H., BINGHAM, B. AND WAKIMOTO, B. T. 1990. The organization and expression of the light gene, a heterochromatic gene of Drosophila melanogaster. Genetics, 125, 129-140.

DOWSETT, A. P. 1983. Closely related species of Drosophila can contain different libraries of middle repetitive DNA sequences. Chromosoma, 88, 104-108.

DOWSETT, A. P. AND YOUNG, M. W. 1982. Differing levels of dispersed repetitive DNA among closely related species of Drosophila. Proc. Natl. Acad. Sci. U.S.A., 79, 4570-4574.

FELGER, I. AND SPERLICH, D. 1989. Cytological localization and organization of dispersed middle repetitive DNA sequences of Drosophila subobscura. Chromosoma, 98, $342-350$.

FONTDEVIla, A., PLA, C., HASSON, E., WASSERMAN, M., SANCHEZ, A., NAVEIRA, H. AND Ruiz, A. 1988. Drosophila koepferae: a new member of the Drosophila serido (Diptera: Drosophilidae) superspecies taxon. Ann. Entomol. Soc. Am., 81, 380-385.

GENETICS COMPUTER GROUP. 1991. Program Manual for the GCG Package. Version 7, April 1991. 575 Science Drive, Madison, WI 53711, U.S.A.

HAMADA, H., PETRINO, M. G. AND KAKUNAGA, T. 1982. A novel repeated element with Z-DNA-forming potential is widely found in evolutionarily diverse eukaryotic genomes. Proc. Natl. Acad. Sci. U.S.A., 79, 6465-6469.

HEALY, M. J., RUSSELL, R. J. AND MIKLOS, G. L. G. 1988. Molecular studies on interspersed repetitive and unique sequences in the region of the complementation group uncoordinated on the $\mathrm{X}$ chromosome of Drosophila melanogaster. Mol. Gen. Genet., 213, 63-71.

HEY, J. 1989. The transposable portion of the genome of Drosophila algonquin is very different from that in $D$. melanogaster. Mol. Biol. Evol., 6, 66-79.

HOCHSTENBACH, R., HARHANGI, H., SCHOUREN, K. AND HENNING, w. 1994. Degenerating Gypsy retrotransposons in a male fertility gene on the Y chromosome of Drosophila hydei. J. Mol. Evol., 39, 452-465.

HUIJSER, P., HENNIG, W. AND DIJKHOF, R. 1987. Poly (dC-dA/dG-dT) repeats in the Drosophila genome: a key function for dosage compensation and position effects? Chromosoma, 95, 209-215.

JACUBCZAK, J. L., XIONG, Y. AND EICKBUSH, T. H. 1990.
Type I (R1) and type II (R2) ribosomal insertions of Drosophila melanogaster are retrotransposable elements closely related to those of Bombyx mori. J. Mol. Biol., 212, 37-52.

JACUBCZAK, J. L., BURKE, W. D. AND EICKBUSH, T. H. 1991. Retrotransposable elements $R 1$ and $R 2$ interrupt the rRNA genes of most insects. Proc. Natl. Acad. Sci. U.S.A., 88, 3295-3299.

KAPLAN, N. L., DARDEN, T. AND LANGLEY, C. H. 1985. Evolution and extinction of transposable elements in Mendelian populations. Genetics, 109, 459-480.

KIM, A., TERZIAN, C., SANTAMARIA, P., PÉlisSON, A., PRUD'HOMME, N. AND BUCHETON, A. 1994. Retroviruses in invertebrates: The gypsy retrotransposon is apparently an infectious retrovirus of Drosophila melanogaster. Proc. Natl. Acad. Sci. U.S.A., 91, 1285-1289.

KIMURA, K., OKUMURA, T., NINAKI, O., KIDWELL, M. G. AND sUZUKI, K. 1993. Transposable elements in commercially useful insects. I. Southern hybridization study of silkworms and honeybees using Drosophila probes. Jap. J. Genet., 68, 63-71.

LANKENAU, D.-H., HUIJSER, P., JANSEN, E., MIEDEMA, K. AND HENNIG, W. 1990. DNA sequence comparison of micropia transposable elements from Drosophila hydei and Drosophila melanogaster. Chromosoma, 99, 111-117.

LINDHOLM D.-A., GUDMUNDSSON, G. H. AND BOMAN, H. G. 1991. a highly repetitive, mariner-like element in the genome of Hyalophora cecropia. J. Biol. Chem., 266, $11518-11521$.

MARÍN, I. AND FONTDEVILA, A. 1995. Characterization of Gandalf, a new inverted-repeat transposable element of Drosophila koepferae. Mol. Gen. Genet., 248, 423-433.

MARÍN, I., LABRADOR, M. AND FONTDEVILA, A. 1992. The evolutionary history of Drosophila buzzatii. XXIII. High content of nonsatellite repetitive DNA in $D$. buzzatii and in its sibling D. koepferae. Genome, 35, 957-964.

MARLOR, R. L., PARKHURST, S. M. AND CORCES, V. G. 1986. The Drosophila melanogaster Gypsy transposable element encodes putative gene products homologous to retroviral proteins. Mol. Cell. Biol., 6, 1129-1134.

MARTIN, G., WIERNASZ, D. AND SCHEDL, P. 1983. Evolution of Drosophila repetitive-dispersed DNA. J. Mol. Evol., 19, 203-213.

MARUYAMA, K. AND HARTL, D. L. 1991. Evidence for interspecific transfer of the transposable element mariner between Drosophila and Zaprionus. J. Mol. Evol., 33, 514-524.

McClURE, M. A. 1991. Evolution of retroposons by acquisition or deletion of retrovirus-like genes. Mol. Biol. Evol., 8, 835-856.

MCCLURE, M. A., JOHNSON, M. S., FENG, D.-F. AND DOOLITTLE, R. F. 1988. Sequence comparisons of retroviral proteins: relative rates of change and general phylogeny. Proc. Natl. Acad. Sci. U.S.A., 85, 2469-2473.

MIKLOS, G. L. G., YAMAMOTO, M.-T., DAVIES, J. AND PIRROTTA, v. 1988. Microcloning reveals a high frequency of repetitive sequences characteristic of chromosome 4 and the $\beta$-heterochromatin of Drosophila melanogaster. Proc. Natl. Acad. Sci. U.S.A., 85, 
2051-2055.

MIZROKHI, L. J. AND MAZO, A. M. 1990. Evidence for horizontal transmission of the mobile element jockey between distant Drosophila species. Proc. Natl. Acad. Sci. U.S.A., 87, 9216-9220.

MiZROKH1, L. J. AND MAZO, A. M. 1991. Cloning and analysis of the mobile element gypsy from D. virilis. Nucl. Acids Res., 19, 913-916.

MORRIS, J., KUSHNER, S. R. AND IVARIE, R. 1986. The simple repeat poly $(\mathrm{dT}-\mathrm{dG})$.poly $(\mathrm{dC}-\mathrm{dA})$ common to eukaryotes is absent from eubacteria and archaebacteria and rare in protozoans. Mol. Biol. Evol., 3, 343-355.

O'NEIL, M. T. AND BELOTE, J. M. 1992. Interspecific comparison of the transformer gene of Drosophila reveals an unusually high degree of evolutionary divergence. Genetics, 131, 113-128.

PARDUE, M. L., LOWENHAUPT, K., RICH, A. AND NORDHEIM, A. 1987. $(\mathrm{dC}-\mathrm{dA})_{\mathrm{n}} \cdot(\mathrm{dG}-\mathrm{dT})_{\mathrm{n}}$ sequences have evolutionarily conserved chromosomal locations in Drosophila with implications for roles in chromosome structure and function. EMBO J., 6, 1781-1789.

PERKINS, H. D. AND HOWELLS, A. J. 1992. Genomic sequences with homology to the $P$ element of Drosophila melanogaster occur in the blowfly Lucilia cuprina. Proc. Natl. Acad. Sci. U.S.A., 89, 10753-10757.

PINNOL, J., FRANCINO, O., FONTDEVILA, A. AND CABRÉ, O. 1988. Rapid isolation of Drosophila high molecular weight DNA to obtain genomic libraries. Nucl. Acids Res., 16, 2736.

RIEDE, 1., JACOB, E. AND RENZ, M. 1983. DNA sequence divergence in the Drosophila virilis group. Chromosoma, 88, 109-115.

ROBERTSON, H. M. 1993. The mariner transposable element is widespread in insects. Nature, 362, 241-245.

RUIZ, A. AND WASSERMAN, M. 1993. Evolutionary cytogenetics of the Drosophila buzzatii species complex. Heredity, 70, 582-596.

RUSSELL, R. J., HEALY, M. J. AND OAKESHOTT, J. G. 1992. Molecular analysis of the lethal (1)B214 region at the base of the $X$ chromosome of Drosophila melanogaster. Chromosoma, 101, 456-466.

SÁNCHEZ, A. 1986. Relaciones Filogenéticas en los Clusters buzzatii $y$ martensis (Grupo repleta) de Drosophila. $\mathrm{Ph}$. D. Thesis, Universitat Autònoma de Barcelona.

SANGER, G., NICKLEN, A. R. AND COULSON, A. R. 1977. DNA sequencing with chain terminating inhibitors. Proc. Natl.
Acad. Sci. U.S.A., 74, 5463-5467.

SHEVELYOV, Y. Y., BALARIKEVA, M. D. AND GVOZDEV, V. A. 1989. Heterochromatic regions in different Drosophila melanogaster stocks contain similar arrangements of moderate repeats with inserted copia-like elements (MDG1). Chromosoma, 98, 117-122.

SILBER, J., BAZIN, C., LEMEUNIER, F., AULARD, S. AND VOLOVITCH, M. 1989. Distribution and conservation of the foldback transposable element in Drosophila. J. Mol. Evol., 28, 220-224.

SMITH, P. A. AND CORCES, v. G. 1991. Drosophila transposable elements: mechanisms of mutagenesis and interactions with the host genome. In: Scandalios, J. G. and Wright, T. R. F (eds) Advances in Genetics, vol. 29., pp. 229-300. Academic Press, San Diego, CA.

SONG, S. U., GERASIMOVA, T., KURKULOS, M., BOEKE, J. D. AND CORCES, V. G. 1994. An Env-like protein encoded by a Drosophila retroelement: evidence that gypsy is an infectious retrovirus. Genes Devel., 8, 2046-2057.

STACEY, S. N., LANSMAN, R. A., BROCK, H. W. AND GR1GL1ATT1, T. A. 1986. Distribution and conservation of mobile elements in the genus Drosophila. Mol. Biol. Evol., 3, 522-534.

TAUTZ, D. AND RENZ, M. 1984. Simple sequences are ubiquitous repetitive components of eukaryotic genomes. Nucl. Acids Res., 12, 4127-4138.

TAUTZ, D., TRICK, M. AND DOVER, G. A. 1986. Cryptic simplicity in DNA is a major source of genetic variation. Nature, 322, 652-656.

VAURY, C., BUCHETON, A. AND PELISSON, A. 1989. The $\beta$ heterochromatic sequences flanking the I elements are themselves defective transposable elements. Chromosoma, 98, 215-224.

wasserman, M. 1992. Cytological evolution of the Drosophila repleta species group. In: Krimbas, C. B. and Powell, J. R (eds) Drosophila Inversion Polymorphism, pp. 455-552. CRC Press, Boca Raton, FL.

WETMUR, J. G. 1991. DNA probes: applications of the principles of nucleic acid hybridization. Crit. Rev. Biochem. Mol. Biol., 26, 227-259.

YounG, M. w. 1979. Middle repetitive DNA: a fluid component of the Drosophila genome. Proc. Natl. Acad. Sci. U.S.A., 76, 6274-6278.

XIONG, Y. AND EICKBUSH, T. H. 1990. Origin and evolution of retroelements based upon their reverse transcriptase sequences. EMBO J., 9, 3353-3362. 